\title{
Elevated serum levels of resistin in inflammatory bowel disease: a meta-analysis
}

\author{
Jixiang Deng ${ }^{1}$, Yuting Chen ${ }^{1}$, Shengqian $\mathrm{Xu}^{2}$, and Faming Pan $^{1}$ \\ ${ }^{1}$ Anhui Medical University \\ ${ }^{2}$ First Affiliated Hospital of Anhui Medical University
}

July 7,2020

\begin{abstract}
The findings of the prior studies investigating the resistin levels in Crohn's disease (CD) patients and ulcerative colitis (UC) patients are inconsistent. Hence we performed the meta-analysis to systematically evaluate the serum levels of resistin in CD and UC, as well as in healthy individuals. PubMed, Web of Science, Embase, Cochrane Library and Chinese National Knowledge Infrastructure (CNKI) (as of April 30, 2020) were searched to collect all relevant published articles. We calculated the pooled standard mean difference (SMD) and 95\% confidence interval (CI) by R software. Eight articles including 438 CD patients and 358 controls, and 9 articles including $391 \mathrm{UC}$ patients and 438 controls were enrolled. The results showed that resistin levels were significantly elevated in patients with $\mathrm{CD}(\mathrm{SMD}=1.76,95 \% \mathrm{CI}: 0.64$ to $2.88, \mathrm{p}=0.002)$ and UC (SMD $=2.10,95 \% \mathrm{CI}$ : 1.03 to $3.17, p<0.001)$ compared with healthy controls. Our findings indicate that the elevated levels of serum resistin were associated with CD and UC.
\end{abstract}

\section{Introduction}

Inflammatory bowel disease (IBD) is a chronic inflammatory condition involving the gut primarily, encompasses two major groups of idiopathic disorders: Crohn's disease (CD) and ulcerative colitis (UC) ${ }^{1}$. Extraintestinal manifestations are also frequently observed and involve the joints, eyes, hepatobiliary tract as well as skin ${ }^{2}$. CD is characterized by transmural inflammation of the intestine. Studies have shown that approximately $50 \%$ of the CD patients have ileocolitis and the incidence is 3.1-20.2 per 100 000. Furthermore, UC is more prevalent than CD with incidence of 9-20 per $10000{ }^{3}$. It starts in the rectum and usually extends proximally through part or the entire colon in a continuous manner ${ }^{4}$. Currently, there is a general agreement that IBD is a result of an interaction of genetic, gut microbiota, intestinal mucosal immune response factors and surrounding environment ${ }^{5}$, and causes suffering, mortality and economic loss every year $^{6}$. Immunopathogenesis of IBD induces an inflammatory response involving both the innate immune cells and the adaptive immune cells ${ }^{7}$. In particular, T-helper 1 and T-helper 2 responses are thought to drive the pathogenesis of $\mathrm{CD}$ and $\mathrm{UC}$, respectively ${ }^{8}$.

Furthermore, adipose tissues can secrete adipokines that play major roles in metabolism, immunity, and inflammation. Meanwhile, resistin is one of the adipokine, mainly produced by macrophages and detectable in human serum. It is a cysteine-rich peptide with different biological effects and initially proposed to regulate obesity, glucose metabolism and insulin sensitivity ${ }^{9}$. Some researchers reported that human resistin may also play a major role in regulating inflammation ${ }^{10}$. Resistin expression has been identified in the nonadipocyte stromal vascular parts of white adipose tissue ${ }^{11}$, moreover, IBD is typically characterized by malnutrition, body composition changes and mesenteric white adipose tissue hypertrophy ${ }^{12}$. And previous studies have investigated the serum levels of resistin in IBD patients and healthy controls ${ }^{13-22}$. However, the results are inconsistent. In light of these inconsistent findings, we made a meta-analysis of published studies that studied serum levels of resistin in patients with CD and UC compared with healthy controls. 


\section{Methods}

\section{Search strategy}

The present meta-analysis was performed according to the Meta-analysis of Observational Studies in Epidemiology (MOOSE) guidelines ${ }^{23}$ and Preferred Reporting Items for Systematic Reviews and Meta-Analyses (PRISMA). We conducted a systematic literature search in PubMed, Web of Science, Embase, Cochrane Library and Chinese National Knowledge Infrastructure (CNKI) databases. The last search was conducted on April 30, 2020. We searched with a combination of keywords, including "adipokines" or "resistin" or "RETN" combined with "Crohn's disease" or "CD" or "ulcerative colitis" or "UC" or "inflammatory bowel disease" or "IBD". The corresponding Chinese terms were applied for CNKI. We also checked other relevant publications on the references of the retrieved studies.

\section{Inclusion criteria and exclusion criteria}

All included studies meet the following criteria: (1) studies published in English or Chinese; (2) studies used a cross-sectional, case - control or clinical cohort design; (3) studies that measured resistin levels in both case and healthy control groups; (4) studies offered sufficient data; and (5) only the most recent or largest sample size study was included for duplicated publications.

The exclusion criteria were as follows: (1) review articles, conference abstracts and case reports; (2) articles did not contain plasma/serum resistin levels of CD or UC patients or healthy controls; (3) duplicate reports or ambiguous data; and (4) studies were not performed in humans.

\section{Quality assessment and data extraction}

Two authors (Jixiang Deng and Yuting Chen) independently screened the manuscripts and extracted data. The Newcastle-Ottawa quality assessment scale (NOS) was performed to assess the methodological quality. The relevant data were extracted from the included studies: first author's name, publication year, study type, study population, sample size, gender, age, body mass index (BMI) and detection methods of resistin levels. Additional, we e-mailed the corresponding author to obtain available information.

\section{Statistical analysis}

For raw data presented as the median and range or the median and interquartile range, the data were transformed to speculate the mean and standard deviation ${ }^{24}$.

The pooled standardized mean difference (SMD) and its $95 \%$ confidence interval (CI) were used to assess the resistin in patients and healthy controls. Moreover, Cochran Q-statistic was applied to evaluate the heterogeneity among the included studies, and $I^{2}$ test was applied to further evaluate the extent of heterogeneity. When heterogeneity existed among studies $\left(p<0.05\right.$ for the $\mathrm{Q}$ test or $\left.I^{2}>50 \%\right)$, a random-effect model was used; otherwise a fixed-effect model was utilized. Meta-regression and subgroup analysis were used to explore the possible sources of heterogeneity. The sensitivity analysis was conducted by removing any single study at a time to assess the reliability of the results. Egger's weighted regression were used to estimate potential publication bias. A two-tailed $p<0.05$ were deemed statistically significant. All statistical analyses were performed by $\mathrm{R}$ (version 3.6.0) software.

\section{Results}

\section{Characteristics of the Included Studies}

We identified a total of 162 articles through database search (Figure 1 presents the flow chart of the study selection process). After screening, 10 articles were finally included. Among them, 8 articles (438 CD patients and 358 healthy controls) and 9 articles (391 UC patients and 438 healthy controls) have studied the serum resistin levels. Table 1 presents the basic characteristics of the enrolled studies.

\section{Results of meta-analysis}


There were significant differences in serum resistin levels between CD patients and healthy controls, and CD patients had higher resistin concentrations $(\mathrm{SMD}=1.76,95 \% \mathrm{CI}=0.64$ to $2.88, p=0.002)$. Meanwhile, serum resistin levels in UC patients were also higher than that in healthy controls (SMD $=2.10,95 \%$ $\mathrm{CI}=1.03$ to $3.17, p<0.001$ ) (Figure 2 ). In addition, subgroup analyses stratified by region showed that resistin levels of CD or UC patients had differences in Europe when compared with healthy controls, whereas no significant differences were found in non-Europe (Figure 3). Meta-regression analysis was applied for detecting additional sources of heterogeneity, indicated that publication year may be a source of heterogeneity across studies (Table 2).

\section{Publication bias and sensitivity analysis}

Egger's test $(t=1.35, p=0.226)$ indicated no significant publication bias was found across the included studies for CD. Meanwhile, Egger's test $(t=1.92, p=0.097)$ also did not find significant publication bias in studies for UC.

Figure 4 shows the outcomes of the sensitivity analysis. The results were not significantly changed after checking the overall effect by the leave one out method, suggesting that the meta-analysis results were stable.

\section{Discussion}

The aim of the present meta-analysis was to investigate possible differences between CD or UC patients and healthy controls in serum resistin levels. The result confirmed that higher resistin levels in CD, UC patients than in healthy individuals. In subgroup analyses stratified by region, we observed that the serum resistin levels of CD and UC patients in Europe were higher, indicating region may be associated with serum resistin concentrations. Moreover, meta-regression for serum resistin levels indicated that publication year may be an explanation for the high heterogeneity across the studies.

Adipokines appear to drive metabolic alterations, pro-inflammatory as well as anti-inflammatory, and human resistin can stimulate the pro-inflammatory cytokines by NF-kappaB (NF- $k$ B) pathway ${ }^{25}$. Resistin was extensively studied in rheumatoid arthritis, ankylosing spondylitis, systemic lupus erythematosus, psoriasis and osteoarthritis ${ }^{26-28}$. It can bind toll-like receptor 4 (TLR4) on human leukocytes and increase the producing of pro-inflammatory cytokines, such as interleukin(IL)-6, IL-12 and IL-1 $\beta$, in turn, these cytokines also enhance resistin expression ${ }^{29}$. It also can stimulate tumor necrosis factor(TNF)-a secretion and activate NF- $k \mathrm{~B}^{25}$. Furthermore, resistin is associated with disease activity, drug use, blood glucose level, glucose tolerance and insulin resistance ${ }^{30,31}$. Taken together, the resistin has been comfirmed to play a crucially role in autoimmune diseases, inflammatory as well as various metabolic.

The causes of the aberrant immune response in IBD patients is unclear, CD might result from the interaction between enetic predisposition, environmental factors, and altered gut microbiota, leading to hyperactive gutassociated immune response ${ }^{32}$. In contrast with $\mathrm{CD}$, the UC inflammation is limited to the colonic mucosa and genetic, dietary, and environmental risk factors may also play an pivotal role in the etiology ${ }^{33}$. IBD patient is typically characterized hypertrophy of the mesenteric adipose tissue and alterations in local or serum adipokine concentrations ${ }^{34}$. Our result confirmed that higher resistin concentrations in CD, UC patients. Although the etiology of IBD remains unkonwn, resistin may act as a new serological biomarkers of IBD.

Subgroup analyses stratified by region showed elevated serum resistin levels in CD and UC patients in the studies conducted in Europe, indicating the presence of regional differences. However, heterogeneity was high in the meta-analysis that compared serum resistin levels between CD and UC patients and healthy controls. Meta-regression and subgroup analysis were performed to detect the potential reasons of heterogeneity. Studies have demonstrated positive relationships between obesity and IBD risk ${ }^{35}$. In meta-regression analysis, year of publication, size of sample, age of cases, BMI of cases were incorporated as covariates, the results suggested that the source of heterogeneity may come from publication year.

To our knowledge, the current study shows the first meta-analysis that combines all published studies and 
presents the differences of resistin concentrations in CD, UC patients. Several limitations deserve to be considered in our study. Firstly, the heterogeneity among studies was high. Secondly, studies evaluating resistin in CD and UC are limited and insufficient data might account for the high heterogeneity among these studies. Future studies examining the resistin concentrations in a larger number of CD and UC patients are required.

In summary, the levels of resistin are significantly elevated in CD and UC patients compared with healthy controls, suggesting a pivotal role of resistin in IBD pathogenesis.

\section{References}

1. Ko, JK, Auyeung, KK. Inflammatory bowel disease: etiology, pathogenesis and current therapy. Curr Pharm Des 2014; 20,1082-96.https://www.doi.org/10.2174/13816128113199990416

2. Greuter, T, Vavricka, SR. Extraintestinal manifestations in inflammatory bowel disease - epidemiology, genetics, and pathogenesis. Expert Rev Gastroenterol Hepatol 2019; 13,30717.https://www.doi.org/10.1080/17474124.2019.1574569

3. Gajendran, M, Loganathan, P, Catinella, AP, Hashash, JG. A comprehensive review and update on Crohn's disease. Dis Mon 2018; 64,20-57.https://www.doi.org/10.1016/j.disamonth.2017.07.001

4. Ordas, I, Eckmann, L, Talamini, M, Baumgart, DC, Sandborn, WJ. Ulcerative colitis. Lancet 2012; 380,1606-19.https://www.doi.org/10.1016/s0140-6736(12)60150-0

5. Fiocchi, C. Inflammatory bowel disease pathogenesis: where are we? J Gastroenterol Hepatol 2015; 30 Suppl 1,12-8.https://www.doi.org/10.1111/jgh.12751

6. Burisch, J, Jess, T, Martinato, M, Lakatos, PL. The burden of inflammatory bowel disease in Europe. J Crohns Colitis 2013; 7,322-37.https://www.doi.org/10.1016/j.crohns.2013.01.010

7. Zundler, S, Neurath, MF. Immunopathogenesis of inflammatory bowel diseases: functional role of T cells and T cell homing. Clin Exp Rheumatol 2015; 33,S19-28.

8. Wallace, KL, Zheng, LB, Kanazawa, Y, Shih, DQ. Immunopathology of inflammatory bowel disease. World J Gastroenterol 2014; 20,6-21.https://www.doi.org/10.3748/wjg.v20.i1.6

9. Park, HK, Kwak, MK, Kim, HJ, Ahima, RS. Linking resistin, inflammation, and cardiometabolic diseases. Korean J Intern Med 2017; 32,239-47.https://www.doi.org/10.3904/kjim.2016.229

10. Jamaluddin, MS, Weakley, SM, Yao, Q, Chen, C. Resistin: functional roles and therapeutic considerations for cardiovascular disease. Br J Pharmacol 2012; 165,622-32.https://www.doi.org/10.1111/j.14765381.2011.01369.x

11. Jung, HS, Park, KH, Cho, YM, Chung, SS, Cho, HJ, Cho, SY, Kim, SJ, Kim, SY, Lee, HK, Park, KS. Resistin is secreted from macrophages in atheromas and promotes atherosclerosis. Cardiovasc Res 2006; 69,76-85.https://www.doi.org/10.1016/j.cardiores.2005.09.015

12. Karmiris, K, Koutroubakis, IE, Kouroumalis, EA. Leptin, adiponectin, resistin, and ghrelin-implications for inflammatory bowel disease. Mol Nutr Food Res 2008; 52,85566.https://www.doi.org/10.1002/mnfr.200700050

13. Trejo-Vazquez, F, Garza-Veloz, I, Villela-Ramirez, GA, et al. Positive association between leptin serum levels and disease activity on endoscopy in inflammatory bowel disease: A case-control study. Exp Ther Med 2018; 15,3336-44.https://www.doi.org/10.3892/etm.2018.5835

14. Frivolt, K, Schwerd, T, Schatz, SB, Freudenberg, F, Prell, C, Werkstetter, KJ, Bufler, P, Koletzko, S. Hyperadiponectinemia During Infliximab Induction Therapy in Pediatric Crohn Disease. J Pediatr Gastroenterol Nutr 2018; 66,915-9.https://www.doi.org/10.1097/mpg.0000000000001876 
15. Theocharidou, E, Balaska, A, Vogiatzis, K, Tellis, CC, Gossios, TD, Athyros, VG, Tselepis, AD, Karagiannis, A. Hypertrophic Mesenteric Adipose Tissue May Play a Role in Atherogenesis in Inflammatory Bowel Diseases. Inflamm Bowel Dis 2016; 22,2206-12.https://www.doi.org/10.1097/mib.0000000000000873

16. Waluga, M, Hartleb, M, Boryczka, G, Kukla, M, Zwirska-Korczala, K. Serum adipokines in inflammatory bowel disease. World J Gastroenterol 2014; 20,6912-7.https://www.doi.org/10.3748/wjg.v20.i22.6912

17. Bostrom, EA, Ekstedt, M, Kechagias, S, Sjowall, C, Bokarewa, MI, Almer, S. Resistin is associated with breach of tolerance and anti-nuclear antibodies in patients with hepatobiliary inflammation. Scand J Immunol 2011; 74,463-70.https://www.doi.org/10.1111/j.1365-3083.2011.02592.x

18. Kader, NA, El-Din, FA, Khatab, EA, NE, EL. Does plasma resistin level have a role in predicting inflammatory bowel disease activity? Indian J Gastroenterol 2010; 29,126-7.

19. Konrad, A, Lehrke, M, Schachinger, V, Seibold, F, Stark, R, Ochsenkuhn, T, Parhofer, KG, Goke, B, Broedl, UC. Resistin is an inflammatory marker of inflammatory bowel disease in humans. Eur J Gastroenterol Hepatol 2007; 19,1070-4.https://www.doi.org/10.1097/MEG.0b013e3282f16251

20. Karmiris, K, Koutroubakis, IE, Xidakis, C, Polychronaki, M, Voudouri, T, Kouroumalis, EA. Circulating levels of leptin, adiponectin, resistin, and ghrelin in inflammatory bowel disease. Inflamm Bowel Dis 2006; 12,100-5.https://www.doi.org/10.1097/01.mib.0000200345.38837.46

21. Abedimanesh, N, Motlagh, B, Abedimanesh, S, Ostadrahimi, A, Somi, MH, Jafarabad, MA, Rezazadeb, M. Circulating resistin in ulcerative colitis, relation with anthropometric, body composition and inflammatory parameters. Progress in Nutrition 2018; 20,132-6.https://www.doi.org/10.23751/pn.v20i2-S.6560

22. Tan, K. The relationship between serum Levels of Resistin, YKL-40 and uIcerative colitis. University of south China 2012.

23. Stroup, DF, Berlin, JA, Morton, SC, Olkin, I, Williamson, GD, Rennie, D, Moher, D, Becker, BJ, Sipe, TA, Thacker, SB. Meta-analysis of observational studies in epidemiology: a proposal for reporting. Meta-analysis Of Observational Studies in Epidemiology (MOOSE) group. Jama 2000; 283,200812.https://www.doi.org/10.1001/jama.283.15.2008

24. Hozo, SP, Djulbegovic, B, Hozo, I. Estimating the mean and variance from the median, range, and the size of a sample. BMC Med Res Methodol 2005; 5,13.https://www.doi.org/10.1186/1471-2288-5-13

25. Silswal, N, Singh, AK, Aruna, B, Mukhopadhyay, S, Ghosh, S, Ehtesham, NZ. Human resistin stimulates the pro-inflammatory cytokines TNF-alpha and IL-12 in macrophages by NF-kappaB-dependent pathway. Biochem Biophys Res Commun 2005; 334,1092-101.https://www.doi.org/10.1016/j.bbrc.2005.06.202

26. Kyriakou, A, Patsatsi, A, Sotiriadis, D, Goulis, DG. Serum Leptin, Resistin, and Adiponectin Concentrations in Psoriasis: A Meta-Analysis of Observational Studies. Dermatology 2017; 233,37889.https://www.doi.org/10.1159/000481882

27. Yang, J, Zhang, X, Ma, Y, et al. Serum levels of leptin, adiponectin and resistin in patients with ankylosing spondylitis: A systematic review and meta-analysis. Int Immunopharmacol 2017; 52,3107.https://www.doi.org/10.1016/j.intimp.2017.09.029

28. Huang, Q, Tao, SS, Zhang, YJ, et al. Serum resistin levels in patients with rheumatoid arthritis and systemic lupus erythematosus: a meta-analysis. Clin Rheumatol 2015; 34,171320.https://www.doi.org/10.1007/s10067-015-2955-5

29. Hutcheson, J. Adipokines influence the inflammatory balance in autoimmunity. Cytokine 2015; 75,2729.https://www.doi.org/10.1016/j.cyto.2015.04.004

30. Tsiotra, PC, Boutati, E, Dimitriadis, G, Raptis, SA. High insulin and leptin increase resistin and inflammatory cytokine production from human mononuclear cells. Biomed Res Int 2013; 2013,487081.https://www.doi.org/10.1155/2013/487081 
31. Acquarone, E, Monacelli, F, Borghi, R, Nencioni, A, Odetti, P. Resistin: A reappraisal. Mech Ageing Dev 2019; 178,46-63.https://www.doi.org/10.1016/j.mad.2019.01.004

32. Torres, J, Mehandru, S, Colombel, JF, Peyrin-Biroulet, L. Crohn's disease. Lancet 2017; 389,174155.https://www.doi.org/10.1016/s0140-6736(16)31711-1

33. Adams, SM, Bornemann, PH. Ulcerative colitis. Am Fam Physician 2013; 87,699-705.

34. Schaffler, A, Scholmerich, J, Buchler, C. Mechanisms of disease: adipocytokines and visceral adipose tissue-emerging role in intestinal and mesenteric diseases. Nat Clin Pract Gastroenterol Hepatol 2005; 2,103-11.https://www.doi.org/10.1038/ncpgasthep0090

35. Kreuter, R, Wankell, M, Ahlenstiel, G, Hebbard, L. The role of obesity in inflammatory bowel disease. Biochim Biophys Acta Mol Basis Dis 2019; 1865,63-72.https://www.doi.org/10.1016/j.bbadis.2018.10.020

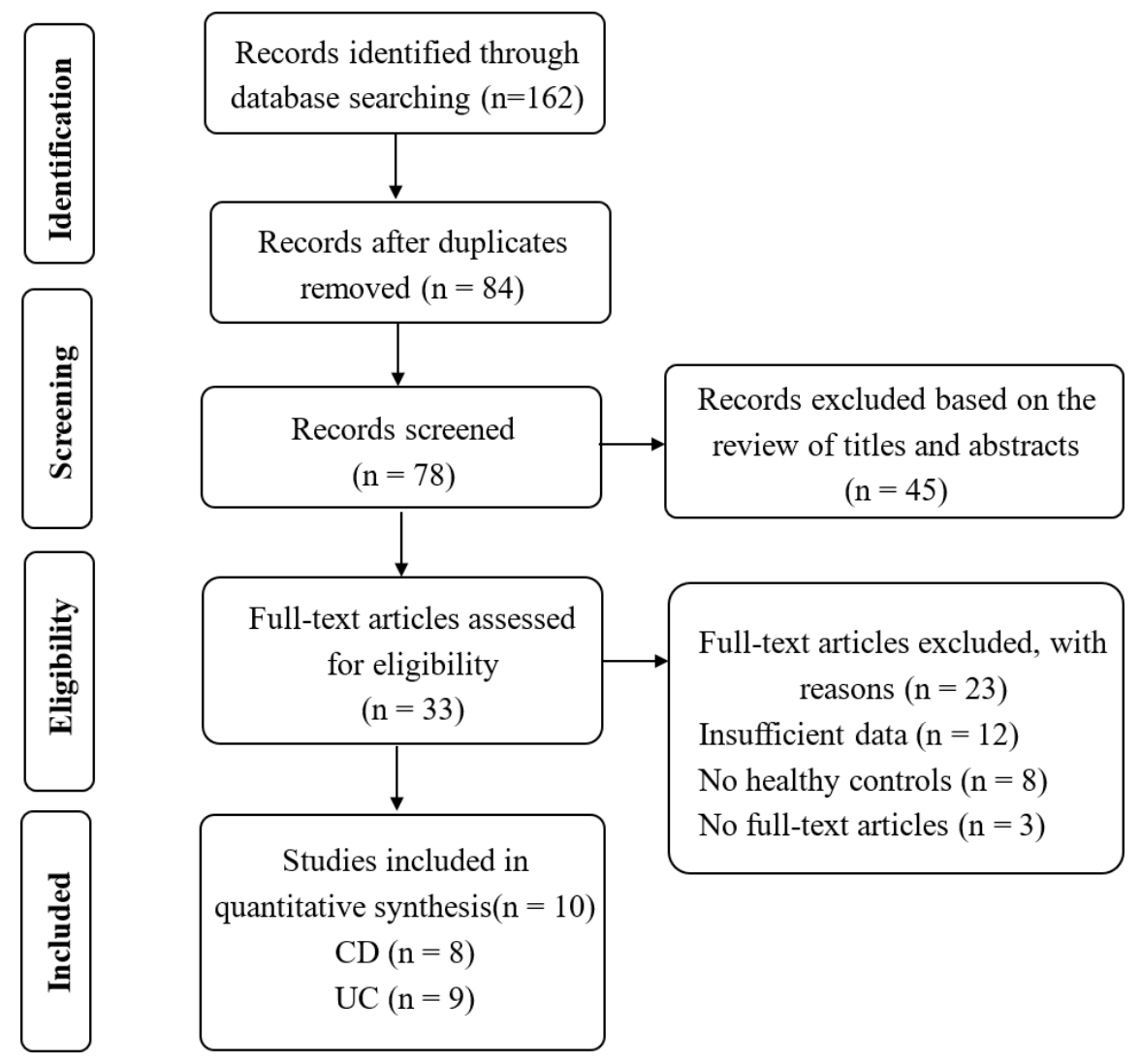


CD

Study

Standardised Mean

Difference

SMD $\quad 95 \%-\mathrm{Cl}$ Weight

Trejo-Vazquez F 2018

Frivolt K 2018

Theocharidou E 2016

Waluga M 2014

Bostrom EA 2011

Kader NA 2010

Konrad A 2007

Karmiris K 2006

Random effects mode

Heterogeneity: $I^{2}=97 \%, \tau^{2}=2.4794, p<0.01$

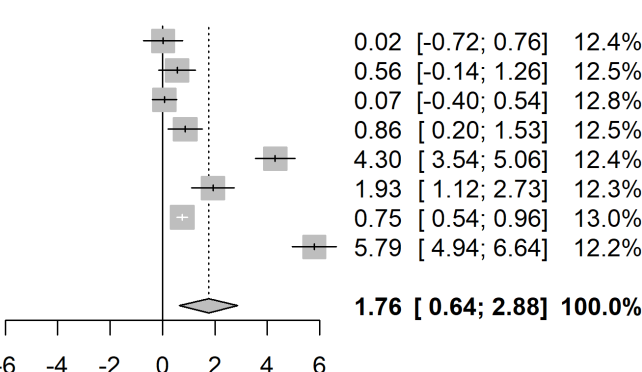

UC

Study

Trejo-Vazquez F 2018

Abedimanesh N 2018

Theocharidou E 2016

Waluga M 2014

Tan K 2012

Bostrom EA 2011

Kader NA 2010

Konrad A 2007

Karmiris K 2006

Random effects mode

Heterogeneity: $I^{2}=97 \%, \tau^{2}=2.5547, p<0.01$

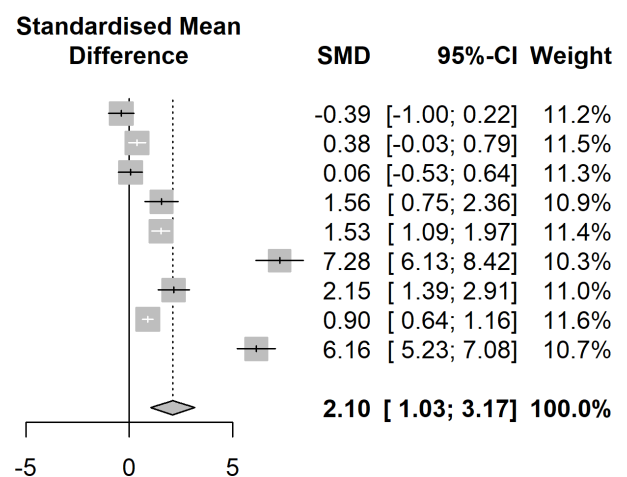


CD

Study

Region $=$ Europe

Frivolt K 2018

Theocharidou E 2016

Waluga M 2014

Bostrom EA 2011

Konrad A 2007

Karmiris K 2006

Random effects model

Heterogeneity: $I^{2}=98 \%, \tau^{2}=2.9466, p<0.01$

Region $=$ Non-Europe

Trejo-Vazquez F 2018

Kader NA 2010

Random effects model

Heterogeneity: $I^{2}=91 \%, \tau^{2}=1.6564, p<0.01$

Random effects mode

Heterogeneity: $I^{2}=97 \%, \tau^{2}=2.4794, p<0.01$

Residual heterogeneity: $I^{2}=97 \%, p<0.01$

UC

Study

Region $=$ Europe

Theocharidou E 2016

Waluga M 2014

Bostrom EA 2011

Konrad A 2007

Karmiris K 2006

Random effects model

Heterogeneity: $I^{2}=98 \%, \tau^{2}=5.9081, p<0.01$

Region $=$ Non-Europe

Trejo-Vazquez F 2018

Abedimanesh N 2018

Tan K 2012

Kader NA 2010

Random effects model

Heterogeneity: $I^{2}=93 \%, \tau^{2}=0.9184, p<0.01$

Random effects model

Heterogeneity: $I^{2}=97 \%, \tau^{2}=2.5547, p<0.01$

Residual heterogeneity: $I^{2}=97 \%, p<0.01$

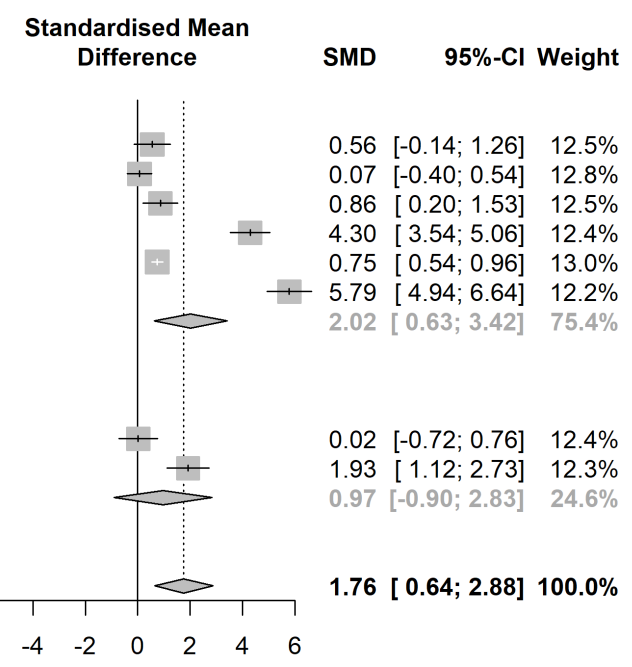

Standardised Mean

Difference

SMD $\quad 95 \%-\mathrm{Cl}$ Weight

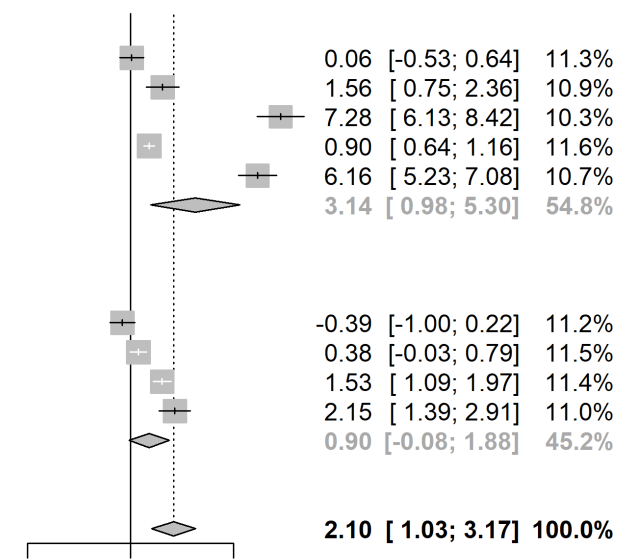

$\begin{array}{lll}-5 & 0 & 5\end{array}$

$2.10[1.03 ; 3.17] 100.0 \%$ 
$C D$

Study

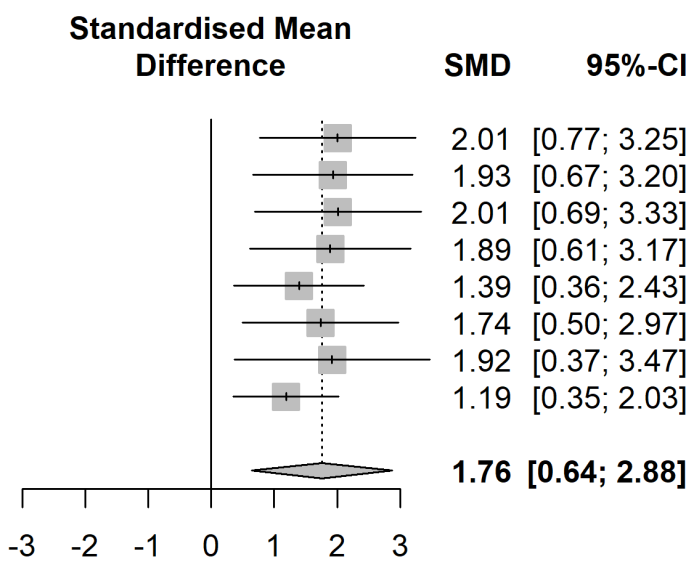

UC

Study

Omitting Trejo-Vazquez F 2018 Omitting Abedimanesh N 2018 Omitting Theocharidou E 2016 Omitting Waluga M 2014 Omitting Tan K 2012 Omitting Bostrom EA 2011 Omitting Kader NA 2010 Omitting Konrad A 2007

Omitting Karmiris K 2006

Random effects model

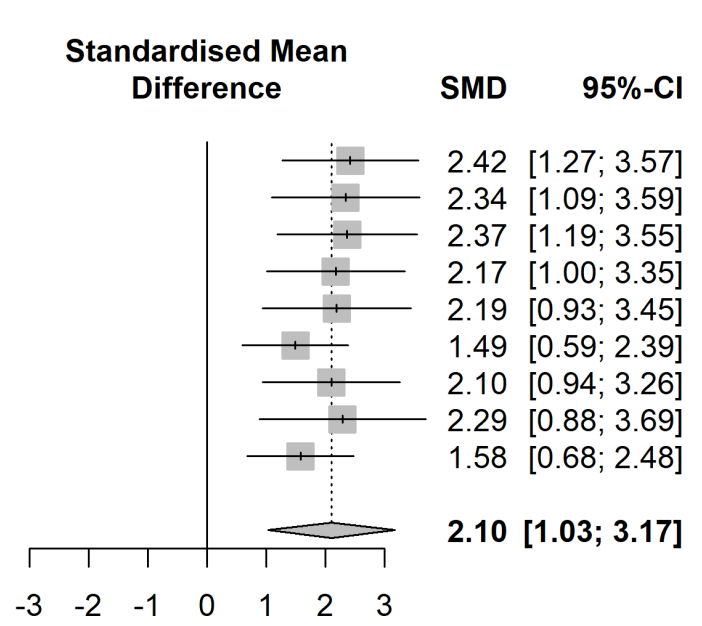

Hosted file

Table 1.docx available at https://authorea.com/users/322255/articles/467313-elevated-serumlevels-of-resistin-in-inflammatory-bowel-disease-a-meta-analysis

Hosted file

Table 2.docx available at https://authorea.com/users/322255/articles/467313-elevated-serumlevels-of-resistin-in-inflammatory-bowel-disease-a-meta-analysis 